\title{
Guest Editorial: Special Section on Enabling Technologies and Methodologies for Knowledge Discovery and Data Mining in Smart Grids
}

T he advance in the research of Smart Grid methodologies ns the doors toward the conceptualization of new tools aimed at effectively addressing most challenging issues of modern power distribution systems, including the massive pervasion of renewable power generators, the strictest power quality limits, the complex interactions with the energy markets, the raising levels of security and reliability constraints, the need for maximizing the exploitation of existing electrical infrastructures [1].

In particular, it is well note that the large-scale integration of distributed and dispersed generators into running electrical grids perturbs the power system operation, inducing a number of complex side-effects mainly related to the stochastic nature of the generated power profiles, and the limited generation hosting capacity of power distribution systems, which have been traditionally designed according to the so called "passivity hypothesis", namely without assuming any generation on the load buses. Hence, the research for advanced tools for properly coordinating the operation of distributed renewable power generators in order to mitigate their negative impacts on power system operation and control, represent a strategic issue to address.

To address this complex issue, paper [2] explores the potential role of microgrids and energy storage systems, which have been considered as the most promising enabling technologies for dealing with an increased penetration of intermittent, and non-programmable generators in electrical distribution systems. In particular, this paper proposes a novel methodology based on particle swarm optimization for storage systems sizing in wind power integrated microgrids. The main idea is to integrate a cost-benefit analysis and here-and- now approach for minimizing the operation costs, maximizing the total system benefit, and considering the wind power uncertainty, for both stand- alone and grid-connected microgrid operation mode. Detailed simulation results on several case studies demonstrated the effectiveness of the proposed methodology, showing that the optimal size of the storage systems and the solution of the unit commitment problem are drastically influenced by the effects of the wind power uncertainty, which requires the deployment of reliable and proactive control frameworks.

The backbone of these new control frameworks is the capability of distributed entities, such as software modules, remote processing units, and pervasive sensor networks, to acquire, process and share data according to fixed time constraints determined by the specific application domain [3]. In this context, the enhancement of the energy management systems, which are traditionally based on low scalable architectures, mixed communication technologies, and legacy proprietary platforms, is one of the main technological challenges to face [4]. In addressing this issue, data heterogeneity, a not relevant issue in traditional power systems measurements, represents a major problem, since the deployment of the metering infrastructures is unlikely to grow over time with the same hardware and software architectures. Pervasive storing and processing of massive data-sets represent further complex issues to address, since the number of grid sensors is expected to increase over several orders of magnitude, and the corresponding data streaming should be promptly processed in order to extract actionable information in useful times [5]. In solving these complex issues, the power system operators must properly represent and discovery the intrinsic semantic of the measured data in order to have a full understanding of the information context, which allows assessing the degree of confidence of the corresponding content [6].

In this context, paper [7] proposes a methodology for feature extraction from massive power quality data, which tries to properly balance the computational efforts and the satisfactory performance of the algorithm in detecting and classifying the electrical disturbances. In particular, to deal with the intrinsic complexities of the disturbances classification problem, this paper conceptualizes a step of feature extraction that may be calculated and analyzed off-line using synthetic waveforms/signals, which are subsequently validated using field measurements.

The idea of converting massive data acquired by pervasive field sensors into high-level information and eventually into actionable intelligence at different application domain could represent a strategic tool in solving further smart grids operation problems. This asks for conceptualizing knowledge discovery frameworks aimed at processing the measured data to provide storage and inference functionalities specifically designed for supporting the smart grid operation [8]. In this context big data analysis for electromechanical dynamics monitoring represents a relevant issue to address. To solve this problem, paper [9] proposes the mathematical derivation, implementation, parameter tuning, and application of a novel data processing algorithm, which aims at identifying instantaneous relationship of system oscillation modes with respect to operating conditions from measured data streams. The proposed algorithm is based on a parallel processed on-line supervised learning algorithm, which integrates two advanced machine-learning algorithms, namely the " $\mathrm{K}$ Nearest Neighbors" and the "Locally Weighted Linear Regression" paradigm, and it has been validated on an 8-generator 36-node system with real operations data.

The advance in research on big data generated from 
pervasive grid sensors is expected to support the evolution of traditional measurement networks toward Wide-Area Monitoring Protection and Control Systems (WAMPACs), which employ a network of time-synchronized sensors, namely the Phasor Measurement Units (PMUs), to implement advance smart grid functions including optimal distributed resource management, smart restoration techniques, and proactive warning services [10]. Field experiences have shown that the pervasive adoption of WAMPACs in smart grids could sensibly reduce the occurrence of large-scale disturbances, by supporting the smart grid operator in implementing advanced protection schemes and adaptive control strategies.

Moreover, the stream of data generated by the PMUs allows simplifying the analysis of the generator dynamics, overcoming the need for identifying an aggregate model of all generators, and allowing the design of load frequency control schemes considering the entire network topology. This feature has been analyzed in paper [11], which proposes a quasidecentralized unscented transform based scheme to control frequency and tie-line power of a multi-area interconnected power system on the basis of dynamic system state estimation based on real-time PMUs measurements. The results presented in this paper demonstrate as the application of this advanced signal-processing algorithm can provide an accurate picture of the actual state of the power system including the dynamic states of generators, which can be utilized in control algorithms to device methods to improve reliable power distribution.

Despite these potential benefits, the development of WAMPACs in power distribution systems is still in its infancy and many open issues are yet to be fixed. A particular challenge is to design holistic and highly flexible WAMPACs architectures, resilient to internal and external disturbances that might compromise their operation. In particular, many papers evidenced that the traditional hierarchical computing architecture is characterized by several shortcomings, which could compromise its deployment in the context of future smart grids.

To fix these issues, many papers emphasized the crucial role played by multi-agent and cooperative paradigms in solving critical smart grid optimization problems, such as optimal management of distributed energy resources, economic dispatch, and demand side management [12-14]. These papers demonstrated that decentralized and selforganizing computing frameworks could sensibly improve the smart grid performances, by mitigating the effect of contingencies, and enhancing the smart grid ability to remain in operation after external disturbances and/or component failures. These benefits have been confirmed in paper [15], where an adaptation of the open shortest path first routing protocol is proposed for on-line optimal network reconfiguration. The proposed algorithm, which has been deployed in secondary substation nodes according to a multi agent-based distributed architecture, has been tested on IEEE 123 modified node test feeder and on a real power distribution system. The obtained results demonstrated the benefits of distributed and decentralized computing paradigms, compared to traditional centralized algorithms in solving complex optimization problems, in terms of scalability and robustness.

On-line smart grids security assessment represents another relevant application domain that would benefit from the development of fast and reliable data-driven optimization techniques [16]. The results of this complex computing process should be obtained according to strictly time constraints, in order to allow the smart grid operator to properly plan preventive and corrective actions aimed at removing or mitigating the effect of critical contingencies. This timeconstrained requirement has stimulated the research for advanced methodologies aimed at proper selecting the list of the most credible contingencies, and at reducing the computational times of the contingency analysis process $[17,18]$.

To address smart girds security analysis, paper [19] conceptualizes a new and efficient security analysis paradigm, which integrates cascading failure simulation module for postcontingency analysis and risk evaluation module based on a decorrelated neural network ensembles algorithm. The proposed algorithm allows to drastically reduce the computational complexities of traditional $\mathrm{N}-\mathrm{k}$ induced cascading contingency analysis, as demonstrated by the simulation results obtained on several realistic case studies.

From the analyzed paper it could be argued that the conceptualization of decentralized, self-organizing, proactive, and holistic computing paradigms aimed at supporting fast decision making in a massive-data, but information-sparse environment represents a very promising research activity. This could stimulate the development of a new generation of computational paradigms aimed at enhancing the smart grid operation procedures with a set of information services for knowledge discovery and data mining. Many important smart grid applications could be benefited from the deployment of these information services, including on-line grid optimization, voltage control, security analysis, synchronized wide-area measurement, pervasive grid monitoring, real-time information sharing, energy price forecasting, and renewable power forecasting.

\section{GUEST EDITORS}

Ahmed Zobaa, Guest Editor

Senior Lecturer

Brunel University London

Uxbridge, London

United Kingdom

e-mail: azobaa@ieee.org

Alfredo Vaccaro, Guest Editor

Associate Professor

University of Sannio

Piazza Roma 21

82100 Benevento, Italy

e-mail: vaccaro@unisannio.it

Loi Lei Lai, Guest Editor

Distinguished Professor

Guangdong University of Technology 
Higher Education Mega Centre

Guangzhou, China

e-mail: 1.1.1ai@ieee.org

\section{ACKNOWLEDGMENT}

The Guest Editors would like to express their deep gratitude to all the authors who have submitted their valuable contributions, and to the numerous and highly qualified anonymous reviewers. We think that the selected contributions, which represent the current state of the art in the field, will be of great interest to the industrial electronics community. They would like to thank Prof. K. Man, the Editor-in-Chief of the IEEE TRANSACTIONS ON INDUSTRIALINFORMATICS, for giving us the opportunity to organize this Special Section and for all the encouragement, help, and support given throughout the process, and L. Pattillo for her professional support and assistance during the whole preparation of this Special Section.

\section{REFERENCES}

[1] V. C. Gungor, D. Sahin, T. Kocak, S. Ergut, C. Buccella, C. Cecati and G. P. Hancke, "Smart Grid Technologies: Communication Technologies and Standards", IEEE Transactions on Industrial Informatics, Vol. 7, No. 4, Nov. 2011, pp. 529-539.

[2] H. Khorramdel, J. Aghaei, B. Khorramdel and P. Siano, "Optimal Battery Sizing in Microgrids Using Probabilistic Unit Commitment"

[3] Q. Yang, J. A. Barria and T. C. Green, "Communication Infrastructures for Distributed Control of Power Distribution Networks," IEEE Transactions on Industrial Informatics, Vol. 7, No. 2, May 2011, pp. 316-327.

[4] A. I. Sabbah, A. El-Mougy and M. Ibnkahla, "A Survey of Networking Challenges and Routing Protocols in Smart Grids", IEEE Transactions on Industrial Informatics, Vol. 10, No. 1, Feb. 2014, pp. 210-221.

[5] C. I. Fan, S. Y. Huang and Y. L. Lai, "Privacy-Enhanced Data Aggregation Scheme Against Internal Attackers in Smart Grid", IEEE Transactions on Industrial Informatics, Vol. 10, No. 1, Feb. 2014, pp. 666-675.

[6] A. Vaccaro , V. Loia , G. Formato, P. Wall and V. Terzija, "A Self Organizing Architecture for Decentralized Smart Microgrids Synchronization, Control and Monitoring", IEEE Transactions on Industrial Informatics, Vol. 11, No. 1, Feb. 2015, pp. 289-298.

[7] F. A. S. Borges, R. A. S. Fernandes, I. N. Silva, and C. B. S. Silva, "Feature Extraction and Power Quality Disturbances Classification Using Smart Meters"

[8] A. Espinoza, M. Ortega, C. Fernandez, J. Garbajosa and A. Alvarez, "Software-intensive systems interoperability in Smart Grids: A semantic approach" Proc. INDIN 2011 9th IEEE International Conference on Industrial Informatics, Lisbon, Portugal, 2011, pp. 739- 744.

[9] J. Zhang, C. Y. Chung, Z. Wang, and X. Zheng, "Instantaneous Electromechanical Dynamics Monitoring in Smart Transmission Grid"

[10] V. Terzija, G. Valverde, D. Cai , P. Regulski, V. Madani, J. Fitch, S. Skok, M.M. Begovic and A. Phadke, "Wide Area Monitoring, Protection and Control of Future Electric Power Networks", Proceedings of the IEEE, Vol.99, No. 1, Jan. 2011, pp. 80-93.

[11] K. Emami, T. Fernando, H. H. C. Iu, B. Nener and K. P. Wong, "Application of Unscented Transform in Frequency Control of a Complex Power System Using Noisy PMU Data"

[12] F. Y. Xu and L. L. Lai, "Novel active time-based demand response for industrial consumers in smart grid," IEEE Transactions on Industrial Informatics, 2015, Digital Object Identifier 10.1109/TII.2015.2446759.

[13] W. Zhang, Y. Xu, W. Liu, C. Zang and H. Yu, "Distributed Online Optimal Energy Management for Smart Grids", IEEE Transactions on Industrial Informatics, Vol. 11, No.3, Jun. 2015, pp.717-727.

[14] A. Safdarian, M. Fotuhi-Firuzabad and M. Lehtonen, "A Distributed Algorithm for Managing Residential Demand
Response in Smart Grids", IEEE Transactions on Industrial Informatics, Vol. 10, No. 4, Nov. 2014, pp. 1551-3203.

[15] F. J. Rodriguez, S. Fernandez, I. Sanz, M. Moranchel, and E. J. Bueno, "Distributed Approach for SmartGrids Reconfiguration based on the OSPF routing Protocol"

[16] J. Navarro, A. Zaballos, A. Sancho-Asensio, G. Ravera and J. E. Armendariz-Inigo, "The Information System of INTEGRIS: INTelligent Electrical GRId Sensor Communications", IEEE Transactions on Industrial Informatics, Vol. 9, No. 3, Aug. 2013, pp. 1548-1560.

[17] K. Anderson, J. Du, A. Narayan and A. El Gamal, "GridSpice: A Distributed Simulation Platform for the Smart Grid", IEEE Transactions on Industrial Informatics, Vol.10, No. 4, Nov. 2014, pp. 2354 - 2363.

[18] J. Navarro, A. Zaballos, A. Sancho-Asensio, G. Ravera and J. E. Armendariz-Inigo, "The Information System of INTEGRIS: INTelligent Electrical GRId Sensor Communications", IEEE Transactions on Industrial Informatics, Vol. 9, No. 3, Aug. 2013, pp. 1548-1560.

[19] Y. Jia, Z. Xu, L. L. Lai, K. P. Wong, "Risk based Power System Security Analysis Considering Cascading Outages"

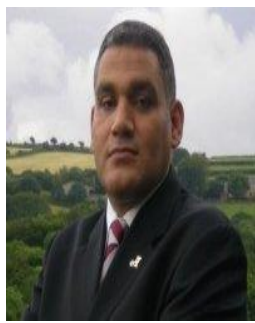

Ahmed Faheem Zobaa (M'02-SM'04) received the B.Sc.(Hons), M.Sc., and Ph.D. degrees in electrical power and machines from Cairo University, Egypt, in 1992, 1997, and 2002, respectively. From 2007 to 2010, he was a Senior Lecturer in renewable energy at University of Exeter, U.K. He was also an Instructor from 1992 to 1997, a Teaching Assistant from 1997 to 2002, an Assistant Professor from 2003 to 2008, an Associate Professor from 2008 to 2013 at Cairo University where he has also been a Professor (on leave) since December 2013. Currently, he is a Senior Lecturer in power systems, an MSc Course Director and a Full Member of the Institute of Energy Futures at Brunel University London, U.K. His main areas of expertise are power quality, (marine) renewable energy, smart grids, energy efficiency, and lighting applications.

Dr. Zobaa is an Editor-in-Chief for the International Journal of Renewable Energy Technology and Technology and Economics of Smart Grids and Sustainable Energy. He is also an Editorial Board member, Editor, Associate Editor, and Editorial Advisory Board member for many international journals. $\mathrm{He}$ is a registered Chartered Engineer, Chartered Energy Engineer, European Engineer, and International Professional Engineer. He is also a registered member of the Engineering Council U.K., Egypt Syndicate of Engineers, and the Egyptian Society of Engineers. He is a Senior Fellow of the Higher Education Academy of U.K. He is a Fellow of the Institution of Engineering and Technology, the Energy Institute of U.K., the Chartered Institution of Building Services Engineers, the Royal Society of Arts, the African Academy of Science, and the Chartered Institute of Educational Assessors. He is a senior member of the Institute of Electrical and Electronics Engineers. Also, he is a member of the International Solar Energy Society, the European Power Electronics and Drives Association, the British Institute of Energy Economics, and the IEEE Standards Association.

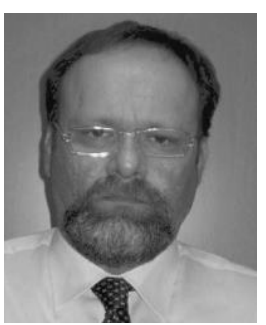

Alfredo Vaccaro received the M.Sc. degree with honors in electronic engineering from the University of Salerno, Salerno, Italy, and the Ph.D. degree in electrical and computer engineering from the University of Waterloo, Ontario, Canada. From 1999 to 2002, he was an Assistant Researcher at the University of Salerno, Department of Electrical and Electronic Engineering. From March 2002 to October 2015, he was an Assistant Professor in electric power systems at the Department of Engineering of the University of Sannio, Benevento, Italy, where he is currently an Associate Professor of electrical Power System. His special fields of interest include soft computing and interval-based method applied to power system analysis, and advanced control architectures for diagnostic and protection of distribution networks. Prof. Vaccaro is a member of the Editorial Boards of IET Renewable Power Generation, and the International Journal of Reliability and Safety, and he is the Executive Editor of the International Journal of Renewable Energy Technology. 


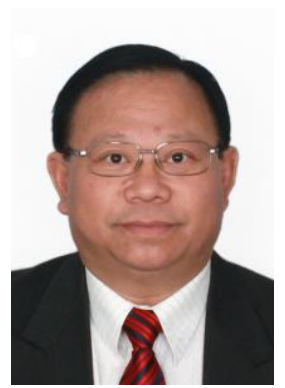

Loi Lei Lai (M'86-SM'92-F'07) received B.Sc. (First Class Honors, the only one), Ph.D. and D.Sc. from University of Aston and City University London respectively. Presently he is a Distinguished Professor at Guangdong University of Technology, Guangzhou China. He was Director of Research and Development Centre, Pao Yue Kong Chair Professor, Vice President and Professor \& Chair in Electrical Engineering for State Grid Energy Research Institute, Beijing China; Zhejiang University, Hangzhou China; IEEE Systems, Man and Cybernetics Society (IEEE/SMCS) and City University London UK respectively. He conducted high-level consultancy for major international projects such as Channel Tunnel between UK and France. In the last few years, he has given 10 keynotes to main international conferences sponsored by IEEE or IET. He was awarded an IEEE Third Millennium Medal, IEEE Power Engineering Society Outstanding Large Chapter Award in 2003, IEEE Power and Energy Society Energy Development and Power Generation Committee Prize Paper in 2006 \& 2009, People of the 2012 Scientific Chinese Prize, IEEE/SMCS Outstanding Contribution Award in 2013 \& 2014 and listed in the honor list of the 2014 The Thousand Talents Plan, China. His research interests are in smart grid, clean energy, computational intelligence applications, big data and signal processing. He is a Fellow of IEEE, IET, Distinguished Expert in State Grid Corporation of China and National Distinguished Expert in China. 